\title{
Oblique Du-Fort Frankel Beam Propagation Method
}

\author{
Ken Chan, Philip Sewell, Ana Vukovic, and Trevor Benson \\ The George Green Institute for Electromagnetic Research, University of Nottingham, Nottingham NG7 2RD, UK
}

Correspondence should be addressed to Ken Chan, eexkc1@nottingham.ac.uk

Received 15 May 2010; Accepted 24 July 2010

Academic Editor: Jun Shibayama

Copyright () 2011 Ken Chan et al. This is an open access article distributed under the Creative Commons Attribution License, which permits unrestricted use, distribution, and reproduction in any medium, provided the original work is properly cited.

The oblique BPM based on the Du-Fort Frankel method is presented. The paper demonstrates the accuracy and the computational improvements of the scheme compared to the oblique BPM based on Crank-Nicholson (CN) scheme.

\section{Introduction}

Increasingly complex optical devices demand computationally fast and memory efficient algorithms for modelling purposes. Finite difference beam propagation method (FDBPM) is a popular numerical technique for simulating large network of optical components due to its computational advantages over classical numerical techniques such as Finite Difference Time Domain (FDTD) method. The BPM method is commonly applied in the Cartesian coordinate system. However when the boundaries of an optical component are not aligned to the Cartesian mesh, for example in the case of tilted waveguides, bends and Mach-Zehnder modulators, sampling on the Cartesian mesh introduces nonphysical staircasing noise. The noise can be minimised by using very fine mesh but that in return incurs large computational costs. To more efficiently reduce the sampling error an improved three-point formulas are used at the interface which take into account the distance between the boundary and the transverse sampling points [1-3]. Further increase in accuracy of the Cartesian BPM, particularly for strongly guided waveguides, is achieved by considering the longitudinal component of magnetic field which is commonly neglected in the standard FD-BPM method [4]. In contrast to Cartesian system, Oblique and Structure Related (SR) coordinate system offers an accurate and efficient alternative for modelling nonorthogonal structures and automatically satisfies $\partial n / \partial z=0$. The sampling grid of the SR mesh is aligned with the component material boundary thus eliminating staircase error and allowing relaxation in mesh size. Various SR-BPM schemes have been introduced [5-11] and different schemes can be combined together to map out the optical component. Furthermore, the SR coordinate system ensures high accuracy for the simple paraxial BPM formulation even without the use of wide-angled schemes [10]. The oblique equation takes into account the propagation direction, which is usually parallel to the structure boundary. Hence the mode-mismatch error is small. One of the motivations for implementation in oblique coordinates is to remove the need for high-order wide-angle scheme which requires substantial computational resources. Wide-angle for oblique coordinate has been developed by Sujecki [11]. However the author has also confirmed that the wide-angle oblique approach should in principle only be applied to low refractive index contrast structures [12].

So far SR and oblique BPM schemes have been implemented using implicit scheme such as $\mathrm{CN}$. Whilst for the two-dimensional (2D) structures this is computationally fast, in the case of modelling three-dimensional (3D) structures the $\mathrm{CN}$ scheme uses iterative matrix solvers such as BI-CGSTAB or GMRES [13] and thus requires huge computational resources. More computationally efficient Alternate Direction Iterative (ADI) schemes [14] cannot be implemented in SR coordinates due to mixed derivatives in transverse directions and are limited to Cartesian meshes. Alternatively, Du-Fort Frankel (DFF) schemes provide larger step size and better stability condition than simple explicit schemes and can be implemented on a parallel computational platform thus providing computational efficiency for modelling realistic 3D optical components. The downside of the DFF scheme is the inherent spurious or "ghost" mode 
that can affect the accuracy and stability of the scheme and which can be alleviated by the right choice of parameters and initial fields $[15,16]$.

In this paper, the oblique BPM method is implemented using the DFF scheme. Section 2 outlines the formulation of the method and Section 3 presents the results for the power loss and computational efficiency of the scheme and compares it against the oblique CN BPM scheme. The results are presented for tilted $2 \mathrm{D}$ and $3 \mathrm{D}$ waveguides and scalar fields.

\section{Formulation}

A general approach for formulating oblique BPM is outlined in [5] and is limited to structures that do not vary with the propagation direction and the $\beta$ reference is parallel to the propagating direction.

In this section an oblique BPM method based on the paraxial approximation and implemented in the DFF algorithm is outlined. Figure 1 shows schematic presentation of two coordinate systems $u, y, z^{\prime}$, and $x, y, z$, where $z$ and $z^{\prime}$ form an angle $\theta$. It is assumed that the fields propagate with respect to the $z^{\prime}$ axis. The $3 \mathrm{D}$ oblique coordinate BPM equation adopted from [9] is used as

$$
\left(\frac{\partial^{2}}{\partial x^{2}}+\frac{\partial^{2}}{\partial y^{2}}+\frac{\partial^{2}}{\partial z^{2}}+k^{2}(x, y, z)\right) \Psi=0
$$

where $\Psi$ represents the scalar field of the form

$$
\Psi\left(u, z^{\prime}\right)=\varphi\left(u, z^{\prime}\right) \exp \left(-j \beta_{0} z^{\prime}\right) .
$$

The relationship between the oblique and the Cartesian coordinate systems is given as

$$
\begin{aligned}
& x=u+z^{\prime} \sin \theta, \\
& z=z^{\prime} \cos \theta .
\end{aligned}
$$

The difference equations are obtained using the chain rule as

$$
\begin{aligned}
& \frac{\partial \Psi}{\partial u}=\frac{\partial \Psi}{\partial x} \\
& \frac{\partial \Psi}{\partial z^{\prime}}=\frac{\partial \Psi}{\partial x} \sin \theta+\frac{\partial \Psi}{\partial z} \cos \theta, \\
& \frac{\partial^{2} \Psi}{\partial u^{2}}=\frac{\partial^{2} \Psi}{\partial x^{2}} \\
& \frac{\partial^{2} \Psi}{\partial u \partial z^{\prime}}=\frac{\partial^{2} \Psi}{\partial x^{2}} \sin \theta+\frac{\partial^{2} \Psi}{\partial x \partial z} \cos \theta, \\
& \frac{\partial^{2} \Psi}{\partial z^{\prime 2}}=\frac{\partial^{2} \Psi}{\partial x^{2}} \sin ^{2} \theta+2 \frac{\partial^{2} \Psi}{\partial x \partial z} \sin \theta \cos \theta+\frac{\partial^{2} \Psi}{\partial z^{2}} \cos ^{2} \theta .
\end{aligned}
$$

Rearranging (6)-(8) and substituting into (1) results in the oblique wave equation:

$$
\left(\frac{\partial^{2} \Psi}{\partial z^{\prime 2}}-2 \frac{\partial^{2} \Psi}{\partial u \partial z^{\prime}} \sin \theta+\frac{\partial^{2} \Psi}{\partial u^{2}}+\frac{\partial^{2}}{\partial y^{2}}\right)+k^{2} \cos \theta \Psi=0
$$

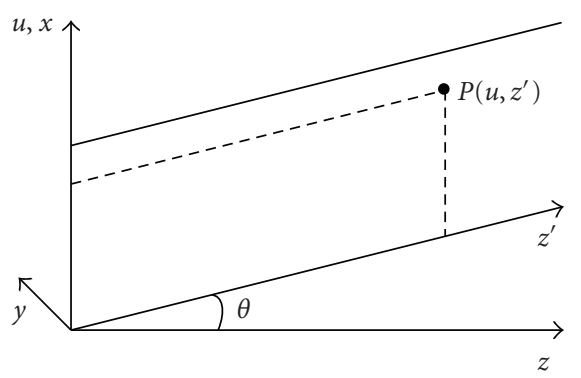

Figure 1: Oblique coordinate system.

Substituting the field from (2) into (9) gives the scalar oblique BPM equation as

$$
\begin{aligned}
2 j \beta_{0} & \frac{\partial \varphi}{\partial z^{\prime}}+\sin \theta \frac{\partial^{2} \varphi}{\partial u \partial z^{\prime}} \\
& =2 j \beta_{0} \sin \theta \frac{\partial \varphi}{\partial u}+\frac{\partial^{2} \varphi}{\partial u^{2}}+\frac{\partial^{2}}{\partial y^{2}}+\left(k^{2} \cos \theta-\beta_{0}^{2}\right) \varphi
\end{aligned}
$$

This equation can be straightforwardly implemented using the $\mathrm{CN}$ scheme. In the case of the 2D modelling where the $\partial^{2} / \partial^{2} y=0, \mathrm{CN}$ scheme requires tridiagonal matrix solver known as Thomas algorithm is used to solve (10) which is much faster than the sparse matrix solver. However in the $3 \mathrm{D}$ modelling, term $\partial^{2} / \partial^{2} y$ in (10) introduces two additional unknown field points in each calculation step thus resulting in five unknown field points. In $\mathrm{CN}$ scheme this requires sparse matrix solver such as the commonly used BI-CGSTAB iterative solver [17].

In order to implement DFF scheme the local field point $\varphi_{l, m, n}$ and the transverse second derivatives are discretized as

$$
\begin{gathered}
\varphi_{l, m, n} \approx \frac{\varphi_{l+1, m, n}+\varphi_{l-1, m, n}}{2} \\
\frac{\partial^{2} \varphi_{l, m, n}}{\partial u^{2}} \approx \frac{\varphi_{l, m+1, n}+\varphi_{l, m-1, n}-\left(\varphi_{l+1, m, n}+\varphi_{l-1, m, n}\right)}{\Delta u^{2}}
\end{gathered}
$$

where $l, m$, and $n$ are the discretised position in the $z^{\prime}$, $u$, and $y$ direction. Substituting the DFF scheme into (10), the numerical implementation for oblique DFF BPM is as follows:

$$
\begin{gathered}
\frac{L}{2}\left(\frac{\varphi_{l+1, m, n}-\varphi_{l-1, m, n}}{\Delta z^{\prime}}-\sin \theta \frac{\varphi_{l, m+1, n}-\varphi_{l, m-1, n}}{2 \Delta u}\right) \\
+\sin \theta\left(\frac{\varphi_{l+1, m+1, n}-\varphi_{l-1, m+1, n}}{2 \Delta u \Delta z^{\prime}}\right. \\
\left.\frac{-\varphi_{l+1, m-1, n}+\varphi_{l-1, m-1, n}}{2 \Delta u \Delta z^{\prime}}\right) \\
=\varphi_{u^{\prime \prime}}+\varphi_{y^{\prime \prime}}+\left(k^{2}-\beta_{0}^{2}\right) \frac{\left(\varphi_{l+1, m, n}+\varphi_{l-1, m, n}\right)}{2}
\end{gathered}
$$

where $L=2 j \beta_{0}$, and $\varphi_{a}^{\prime \prime}=\left(\varphi_{m, a+1}+\varphi_{m, a-1}-\varphi_{m+1, a}-\right.$ $\left.\varphi_{m-1, a}\right) / \Delta a^{2}$.The additional term in (10) diminishes the explicit nature of the DFF, the 3D oblique DFF scheme corresponds to separable tri-diagonal matrices on each layer of the $3 \mathrm{D}$ structure. This can be efficiently solved by 


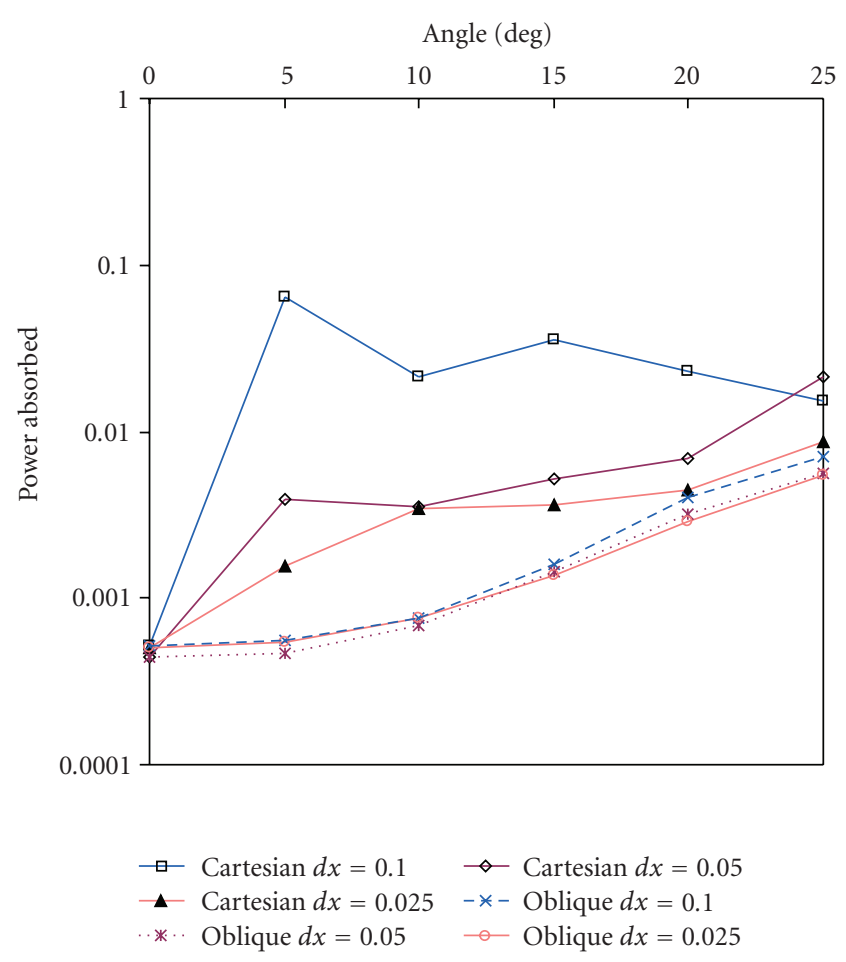

FIgUre 2: Power loss for a range of tilted angles for the oblique DFFBPM and the Cartesian DFF-BPM method.

Thomas algorithm and is algorithmically efficient for parallel computing. Solving of the tri-diagonal matrix is more computationally efficient than solving the sparse matrix thus ensuring better computational efficiency of the $3 \mathrm{D}$ oblique DFF-BPM compared to the oblique CN-BPM.

\section{Results}

In this section the accuracy and computational stability of the oblique DFF-BPM method is analyzed and compared with the oblique CN-BPM method. Both 2D and 3D tilted waveguides are analyzed.

In an oblique coordinate system a tilted waveguide is essentially a straight waveguide and ideally the power transmitted in a tilted waveguide in the oblique system is identical to the power transmitted in a straight waveguide in the Cartesian mesh. Figure 2 compares power loss of the 2D tilted waveguide analyzed using the oblique DFF-BPM with that analyzed using the DFF scheme in Cartesian mesh for different tilted angles and different sampling meshes. Perfectly matched layer (PML) is used for absorbing any leakage from the simulation window. The implementation of PML for the DFF method is described in [16]. The PML layer is set as $1.0 \mu \mathrm{m}$ and strength, $\sigma / \omega$ is set as 10 . Guided mode at the wavelength of $1.15 \mu \mathrm{m}$ is launched in the $1 \mu \mathrm{m}$ wide slab waveguide with core refractive index $n=1$. 1 surrounded by air. Longitudinal sampling $\Delta z^{\prime}$ is fixed at $0.1 \mu \mathrm{m}$ and the waveguide length is $409.6 \mu \mathrm{m}$. It can be seen that by reducing the sampling of the mesh the power loss in the Cartesian $\mathrm{BPM}$ is reduced whilst for the oblique DFF-BPM the change

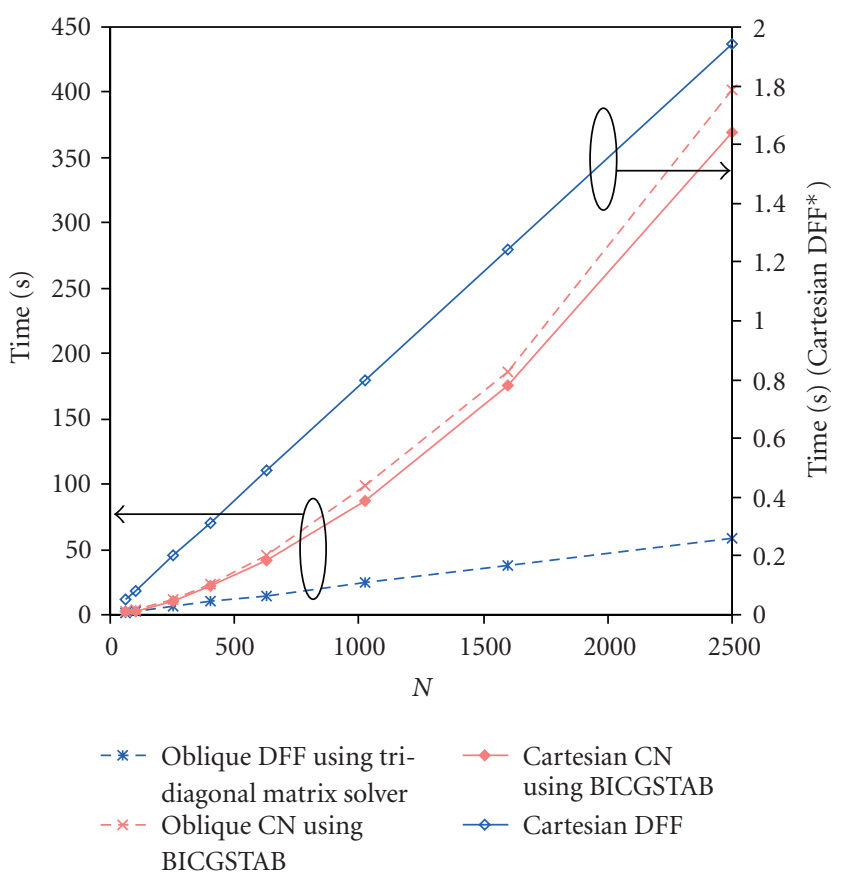

FIgURE 3: Comparison of the computational time for the oblique and Cartesian DFF-BPM and CN-BPM w.r.t. the total number of mesh points $N$.

of the mesh size does not significantly change the power loss. This indicates that the mesh size can be more relaxed in the case of the oblique DFF-BPM ensuring faster run time.

Figure 3 compares the computational runtime of the 3D oblique and Cartesian DFF- and CN-BPM methods for different number of total mesh points $N$. A simple $1 \mu \mathrm{m}$ by $1 \mu \mathrm{m}$ square metal-air waveguide is chosen for a fair speed test. Longitudinal step $\Delta z^{\prime}$ is fixed at $0.05 \mu \mathrm{m}, \Delta u=$ $\Delta y$, and the waveguide is $100 \mu \mathrm{m}$ long. For the oblique simulation, the same waveguide is used but tilted at an angle of $5^{\circ}$. Figure 3 shows that the Cartesian DFF excels in speed even without any parallelization involved. Figure 3 also shows that oblique DFF-BPM is substantially slower than Cartesian DFF-BPM due to the implementation of the Thomas algorithm. However, when compared with the Cartesian and oblique CN-BPM methods, the oblique DFFBPM method is much faster, especially for large mesh sizes. However, it should be noted that DFF-BPM requires smaller longitudinal step size than CN-BPM to achieve the same level of accuracy and maintain stability. The 3D oblique DFF-BPM is also suitable for parallel computing platform allowing for more computationally efficient simulations.

Figure 4 analyses the stability of the oblique DFF-BPM method. It is well known that the main weakness of the DFF algorithm is the appearance of the spurious solution [15]. The position of the spurious mode can be controlled by appropriate choice of the mesh size and the excitation [16]. It is desirable that the spurious mode is not too close to the waveguide true mode so that the stability of the algorithm is not affected. Generally increasing the transverse 


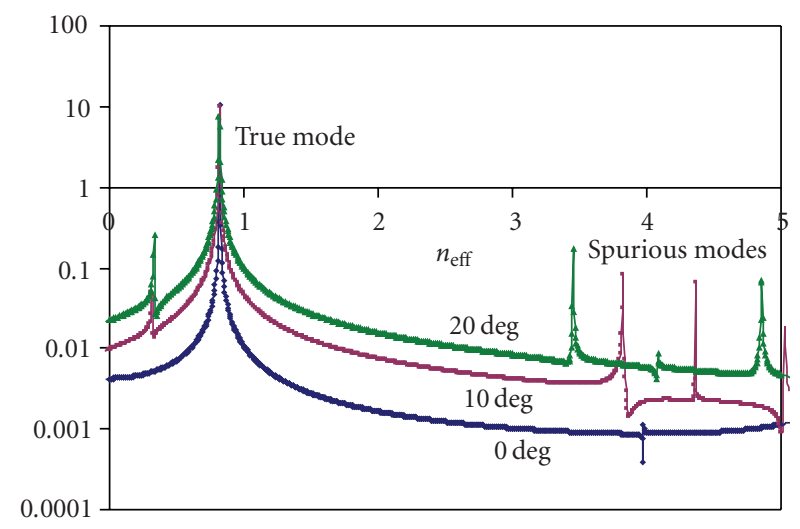

FIGURE 4: Fourier transform of field overlaps along the waveguide to identify the true mode $\left(n_{\text {eff }}=0.82\right)$ and the appearance of spurious modes at various tilted angles.

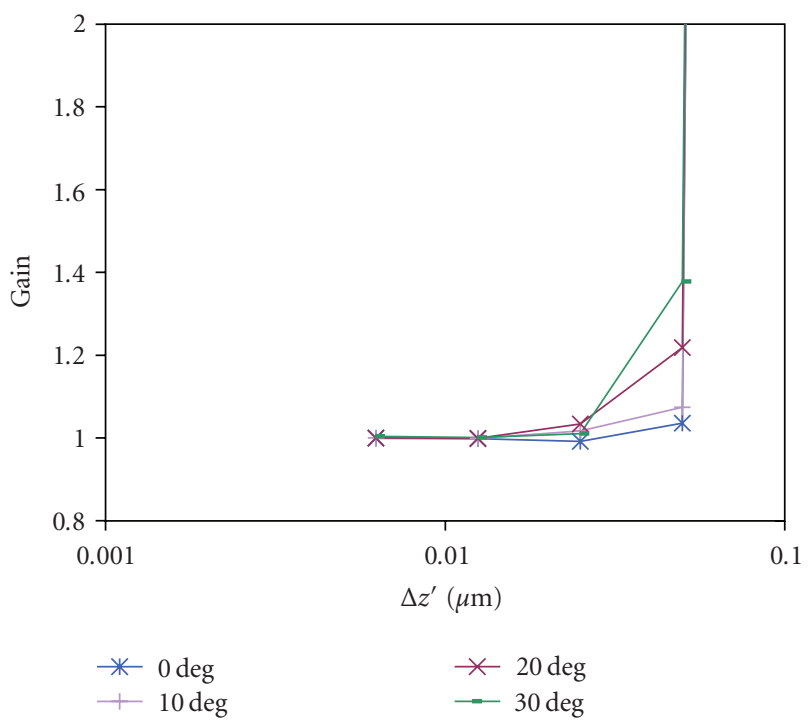

Figure 5: Power gain for oblique DFF. It shows the necessity for keeping the propagation step size small to maintain stability when transverse mesh size is small $(\Delta u=0.01 \mu \mathrm{m})$.

mesh size and reducing the propagation size step will place the spurious mode further away from the true mode [16]. Figure 4 investigates the impact of the tilted angle on the position of the spurious mode for a fixed mesh size. Figure 4 gives the Fourier transform of the field overlap between the incident field and the field evolved along the waveguide for different tilted angles. The 3D waveguide is a rectangular metal-air waveguide with dimensions of $1 \mu \mathrm{m}$ by $0.5 \mu \mathrm{m}$ and $204.8 \mu \mathrm{m}$ long. Transverse mesh size is $\Delta u=\Delta y=$ $0.1 \mu \mathrm{m}$ and longitudinal step sized is $\Delta z^{\prime}=0.05 \mu \mathrm{m}$. Half sine wave of $1.15 \mu \mathrm{m}$ wavelength is launched at the input. Figure 4 shows that the increase of the tilted angle brings the spurious and the true mode closer together. This will have implications on the maximum tilted angle that can be considered using the oblique DFF-BPM method.

The stability of oblique DFF is determined by the oblique angle and the mesh size. The effect of the mesh size is

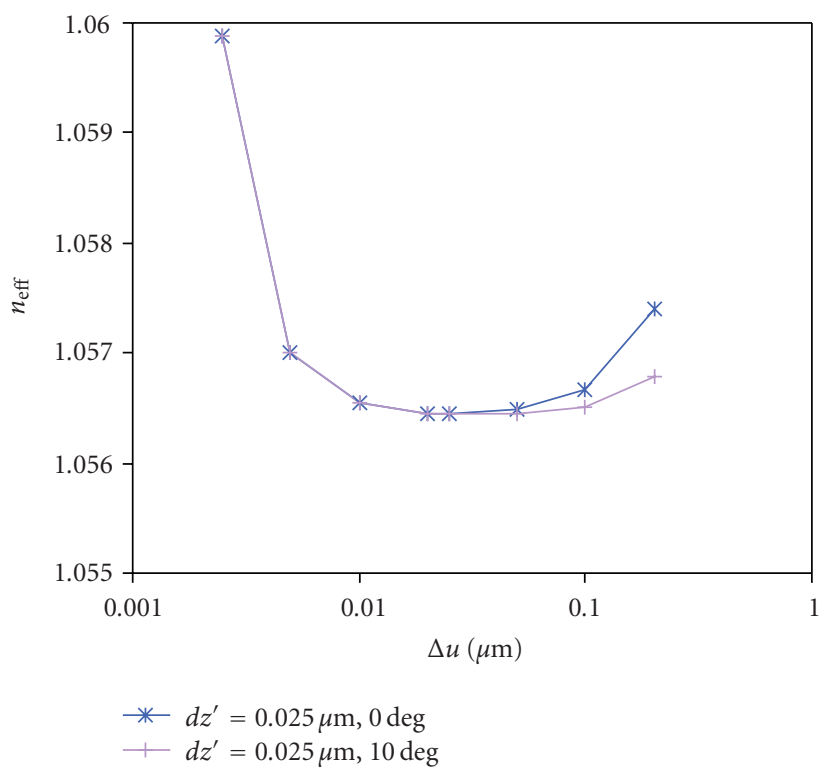

FIGURE 6: Effective index of a slab waveguide using Cartesian DFF and 10 degree tilted oblique DFF method. Accuracy deteriorates as it reaches the unstable region.

examined in Figure 5 for $\Delta u=0.01 \mu \mathrm{m}$ and different tilted angles. The waveguide parameters are as in Figure 2. It shows that for small transverse mesh size, it is necessary to keep the propagation step size small to maintain stability. It can be seen that when the oblique angle is small, the oblique DFF behaves similar to the Cartesian DFF. As the oblique angle increases, the oblique DFF requires smaller propagation step or larger transverse mesh size to maintain the stability.

Figure 6 examines the parameter choice and instability on the calculation of the effective index. The waveguide parameters are the same as in Figure 2. The obtained effective index is plotted for $\Delta z^{\prime}=0.025 \mu \mathrm{m}$ with various mesh sizes and compared between the Cartesian DFF applied to the straight waveguides and the oblique DFF applied to the $10^{\circ}$ tilted waveguide. Figure 6 shows the Cartesian DFF and the $10^{\circ}$ tilted oblique DFF agree very well for small mesh sizes but have significant discrepancy when the transverse mesh size is increased. It can be concluded that the stability condition has restrained the use of very small transverse mesh size in DFF. However, using large transverse mesh size would risk losing accuracy. However, it should be noted that the difference in coordinate system makes it difficult to compare results directly. A slice in the oblique coordinate is equivalent to a diagonal cross-section through multiple slices on a Cartesian coordinate system.

Figures $7(a)-7(\mathrm{c})$ shows the field profiles of the $2 \mathrm{D}$ tilted waveguide obtained using the oblique and Cartesian DFFBPM method. The slab waveguide is as in Figure 2 but $10 \mu \mathrm{m}$ long and tilted by $10^{\circ}$. Figure 7 (a) shows the field profile of the $10^{\circ}$ tilted waveguide mapped in the Cartesian mesh and Figure 7(b) shows the same waveguide analysed using the oblique DFF-BPM method. It can be seen that the Cartesian mesh introduces large staircasing noise even for very fine sampling mesh. For comparison, the field profile 


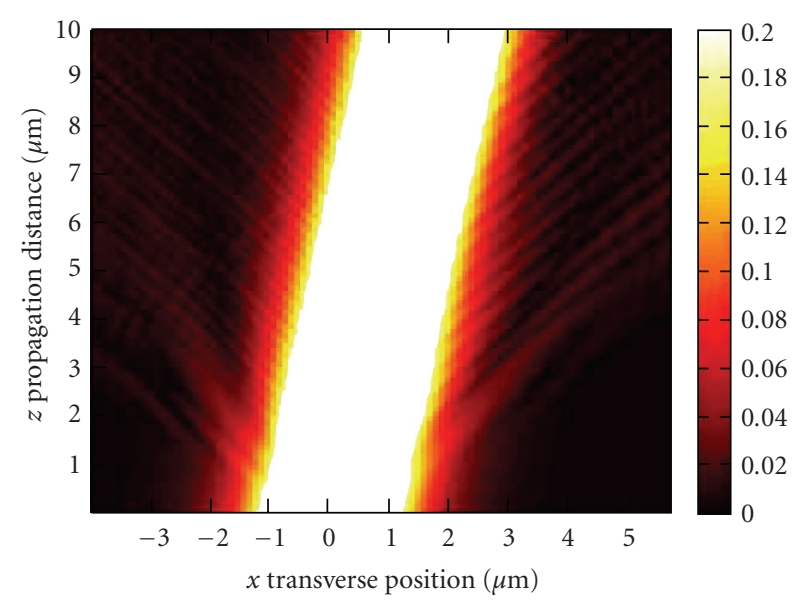

(a)

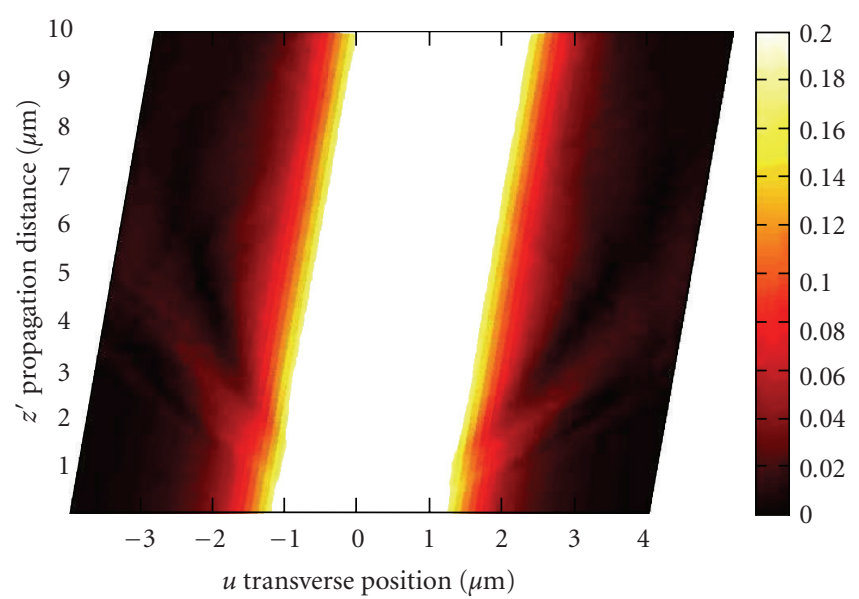

(b)

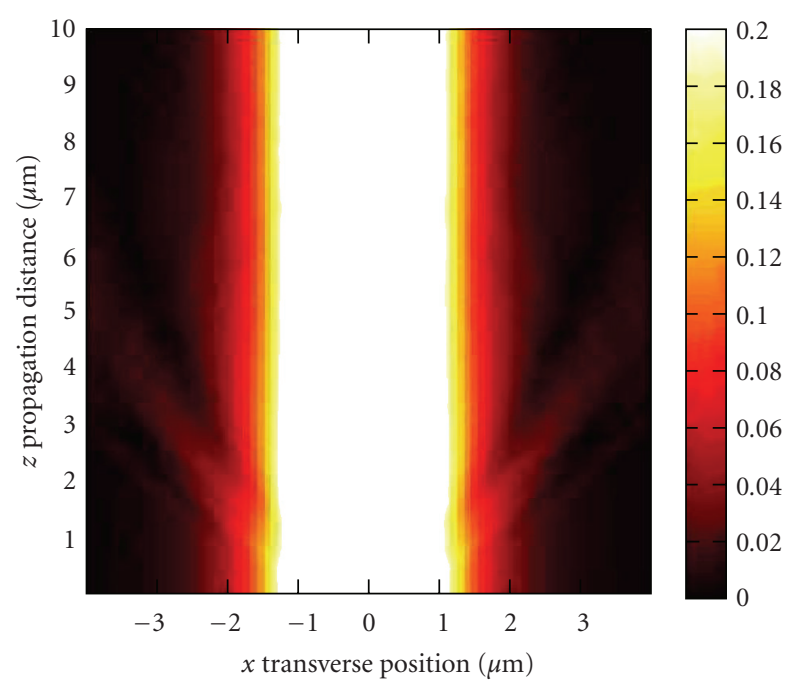

(c)

Figure 7: (a) Field surface plot for $10^{\circ}$ tilted waveguide on Cartesian mesh. Scale has been capped to show the stair case noise. (b) Field surface plot for $10^{\circ}$ tilted waveguide on oblique coordinate. (c) Field surface plot for straight waveguide on Cartesian mesh.

of a standard nontilted waveguide modelled on the Cartesian mesh in Figure 7(c) behaves similarly to the tilted waveguide modelled on oblique coordinate. Perfectly Matched Layer (PML) boundary condition is used to absorb the wave leakage from the waveguide and the nonphysical staircase noise. The slight leakage of the guided mode near the input of the waveguide is due to the mismatch of the analytical mode used to excite the waveguide and the actual numerical field solution. This leakage disappears as the propagating mode is settled in the waveguide.

\section{Conclusion}

The paper demonstrates the implementation of the DFF algorithm into the 3D scalar oblique BPM method. The accuracy and stability of the oblique DFF is investigated on the $2 \mathrm{D}$ and $3 \mathrm{D}$ tilted waveguides and compared against the oblique CN-BPM method. The resulting method is computationally faster than oblique CN-BPM method and is suitable for parallel computing for further computational savings. It is noted that the appearance of the spurious mode can potentially limit the application of the DFF to very large titled angles.

\section{References}

[1] C. Vassallo, "Interest of improved three-point formulas for finite-difference modeling of optical devices," Journal of the Optical Society of America A, vol. 14, no. 12, pp. 3273-3284, 1997.

[2] Y.-P. Chiou, Y.-C. Chiang, and H.-C. Chang, "Improved threepoint formulas considering the interface conditions in the 
finite-difference analysis of step-index optical devices," Journal of Lightwave Technology, vol. 18, no. 2, pp. 243-251, 2000.

[3] Y.-C. Chiang, Y.-P. Chiou, and H.-C. Chang, "Improved fullvectorial finite-difference mode solver for optical waveguides with step-index profiles," Journal of Lightwave Technology, vol. 20, no. 8, pp. 1609-1618, 2002.

[4] J. Yamauchi, Y. Nito, and H. Nakano, "A modified semivectorial BPM retaining the effects of the longitudinal field component and its application to the design of a spot-size converter," Journal of Lightwave Technology, vol. 27, no. 13, pp. 2470-2476, 2009.

[5] T. M. Benson, P. Sewell, S. Sujecki, and P. C. Kendall, "Structure related beam propagation," Optical and Quantum Electronics, vol. 31, no. 9, pp. 689-703, 1999.

[6] D. Z. Djurdjevic, T. M. Benson, P. Sewell, and A. Vukovic, "Fast and accurate analysis of 3-D curved optical waveguide couplers," Journal of Lightwave Technology, vol. 22, no. 10, pp. 2333-2340, 2004.

[7] S. Sujecki, P. Sewell, T. M. Benson, and P. C. Kendall, "Novel beam propagation algorithms for tapered optical structures," Journal of Lightwave Technology, vol. 17, no. 11, pp. 2379-2388, 1999.

[8] G. R. Hadley, "Slanted-wall beam propagation," Journal of Lightwave Technology, vol. 25, no. 9, pp. 2367-2375, 2007.

[9] J. Yamauchi, J. Shibayama, and H. Nakano, "Finite-difference beam propagation method using the oblique coordinate system," Electronics and Communications in Japan, Part II: Electronics, vol. 78, no. 6, pp. 20-27, 1995.

[10] P. Sewell, T. M. Benson, T. Anada, and P. C. Kendall, "Bioblique propagation analysis of symmetric and asymmetric $\mathrm{Y}$ junctions," Journal of Lightwave Technology, vol. 15, no. 4, pp. 688-696, 1997.

[11] S. Sujecki, "Wide-angle, finite-difference beam propagation in oblique coordinate system," Journal of the Optical Society of America A, vol. 25, no. 1, pp. 138-145, 2008.

[12] S. Sujecki, "Generalized rectangular finite difference beam propagation method," Applied Optics, vol. 47, no. 23, pp. 4280-4286, 2008.

[13] H. A. van der Vorst, "Bi-CGSTAB: a fast and smoothly converging variant of Bi-CG for the solution of nonsymmetric linear systems," SIAM Journal on Scientific Computing, vol. 13, no. 2, pp. 631-644, 1992.

[14] D. W. Peaceman and H. H. Rachford, "The numerical solution of parabolic and elliptic differential equations," Journal of the Society for Industrial and Applied Mathematics, vol. 3, p. 28, 1955.

[15] H. M. Masoudi and J. M. Arnold, "Spurious modes in the DuFort-Frankel finite-difference beam propagation method," IEEE Photonics Technology Letters, vol. 9, no. 10, pp. 13821384, 1997.

[16] P. Sewell, T. M. Benson, and A. Vukovic, "A stable DuFortFrankel Beam-Propagation method for lossy structures and those with perfectly matched layers," Journal of Lightwave Technology, vol. 23, no. 1, pp. 374-381, 2005.

[17] S. Sujecki, T. M. Benson, P. Sewell, and P. C. Kendall, "Novel vectorial analysis of optical waveguides," Journal of Lightwave Technology, vol. 16, no. 7, pp. 1329-1335, 1998. 

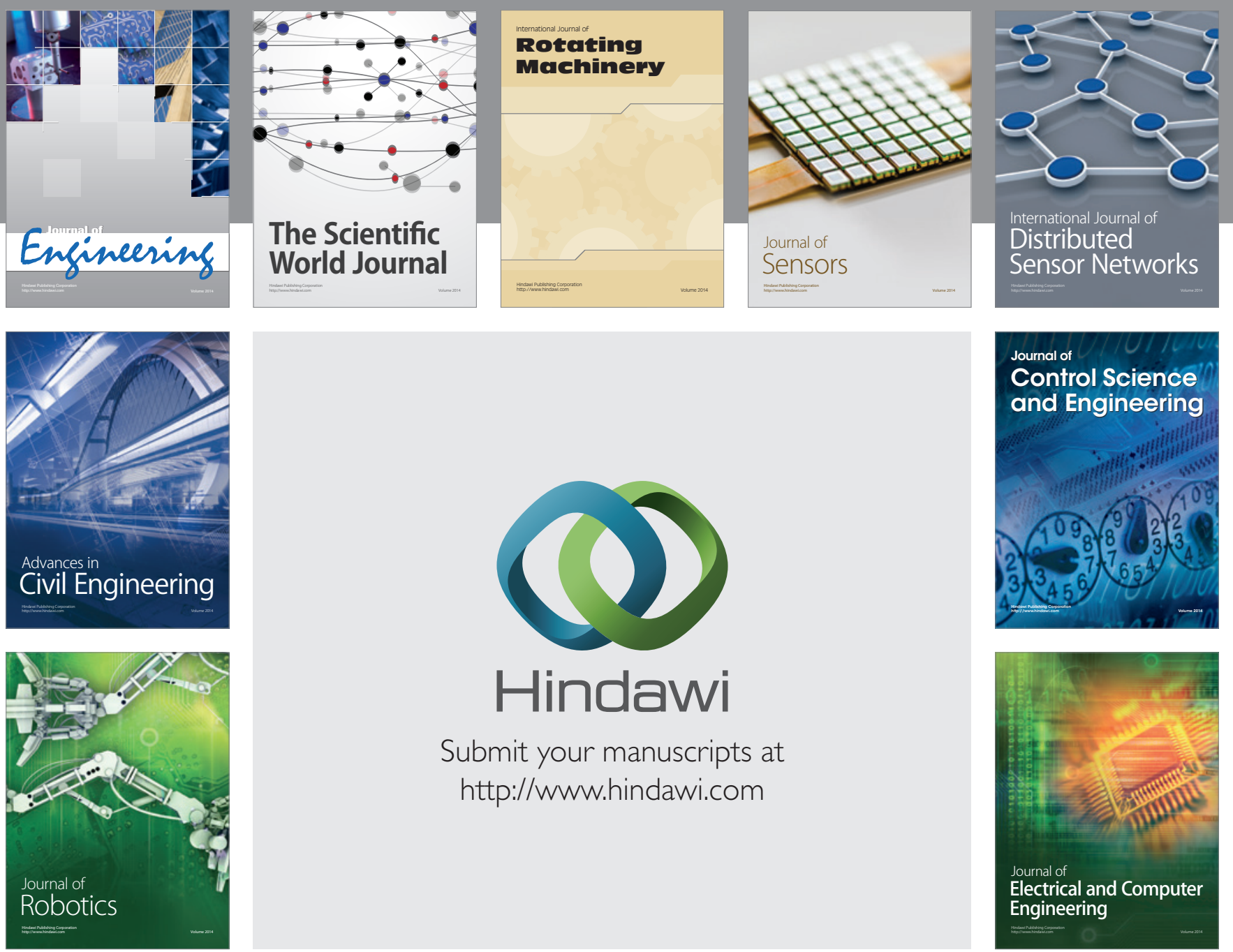

Submit your manuscripts at

http://www.hindawi.com
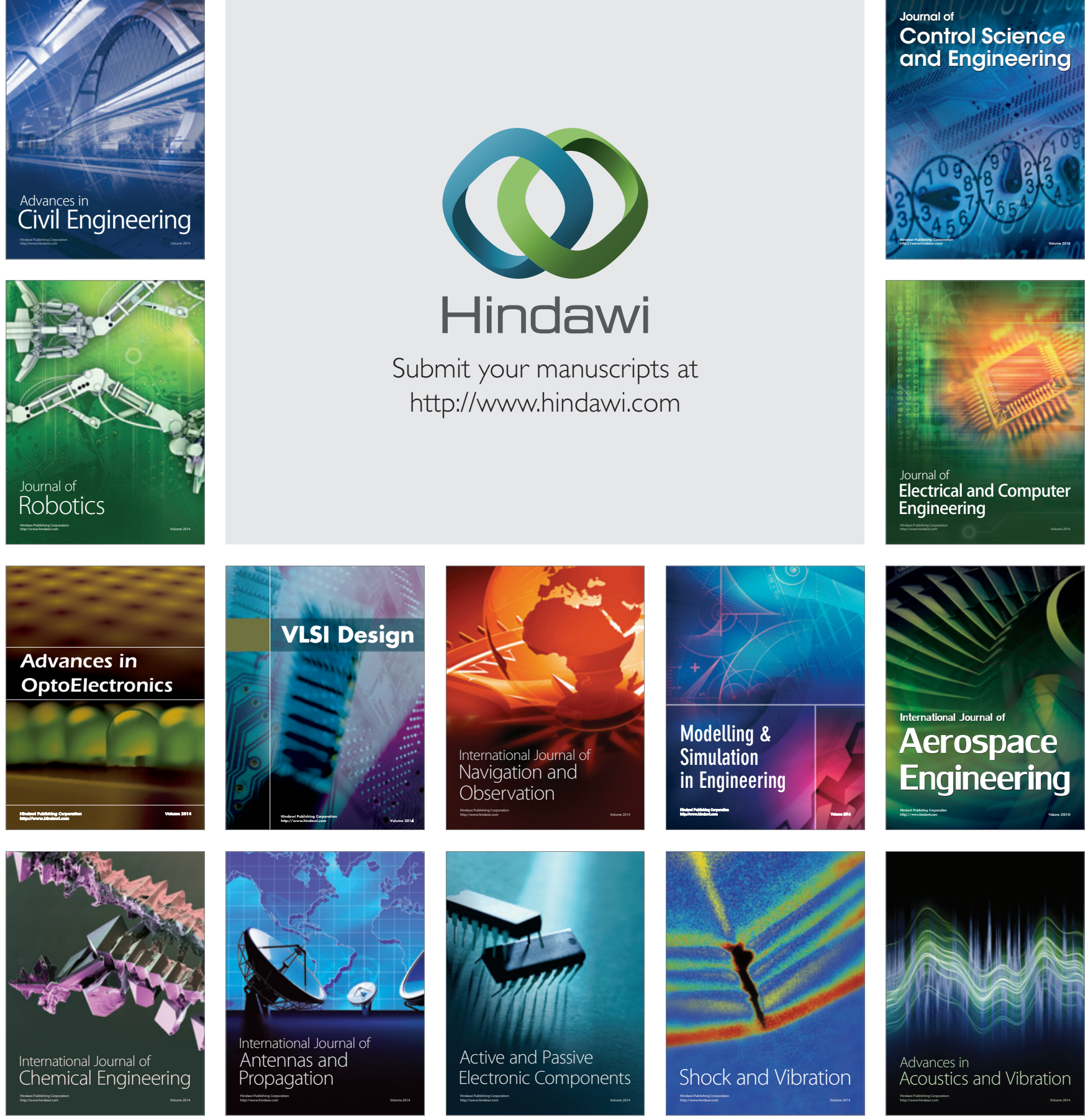